\title{
Microstructure and Orientation Distribution of Aragonite Crystals in Nacreous Layer of Pearl Shells
}

\author{
Kyosuke Yoshimi ${ }^{1,2}$, Mayumi Shoji ${ }^{1,2}$, Tomohisa Ogawa ${ }^{2,3}$, Akira Yamauchi ${ }^{1,2}$, \\ Takako Naganuma $^{3}$, Koji Muramoto ${ }^{3}$ and Shuji Hanada ${ }^{1}$ \\ ${ }^{1}$ Institute for Materials Research, Tohoku University, Sendai 980-8577, Japan \\ ${ }^{2}$ Center for Interdisciplinary Research, Tohoku University, Sendai 980-8578, Japan \\ ${ }^{3}$ Department Biomolecular Science, Tohoku University, Sendai 981-8555, Japan
}

\begin{abstract}
The microstructure and orientation distribution of aragonite crystals in the nacreous layer of cultured Pteria penguin are investigated in this paper. Helical patterns formed by growth forefronts of the nacreous layer for good-quality shells are observed using a laser microscope, whereas no clear pattern is observed on the nacreous layer surfaces of bad-quality shells. The observed aragonite crystals are plate-shaped and hexagonal for both the good and bad-quality shells, in which the top and bottom faces are parallel to the (001) plane and the side faces are parallel to the $\{110\}$ and (010) planes. The aragonite crystals in the nacreous layer of good-quality shells seem to be harmonically oriented along a crystallographic direction. These orientation distributions basically indicate that the (001) basal planes are parallel to the inner shell surface, and the [100] and [010] axes are oriented in almost the same direction, respectively. Some of the aragonite crystals are rotated about the $c$ axis by approximately $\pm 60^{\circ}$ from the basic orientation distribution. On the other hand, the aragonite crystals of bad-quality shells seem to be randomly oriented in the nacreous layer. These orientation distributions indicate that the (001) basal planes are parallel to the inner shell surface in a similar manner as those of good-quality shells, but their [100] and [010] axes are randomly oriented about the $c$ axis. Therefore, it is considered that such different orientation distributions result in different qualities of pearls that are developed in the shells.
\end{abstract}

(Received November 20, 2003; Accepted March 26, 2004)

Keywords: biomineralization, pearl, aragonite, nacreous layer, texture

\section{Introduction}

Minerals that are produced biologically and precipitated in vivo and/or in vitro are called "biominerals". These comprise crystalline as well as amorphous matter. In the case of crystalline biominerals, the crystals are further assembled with each other and eventually develop into hard tissues. Bones, teeth, shells, and so on are typical hard tissues produced by so-called "biomineralization". For instance, bones and teeth consist of calcium phosphates and shells consist of calcium carbonates. In hard tissues, some proteins exist between the crystals and function as binding agents. Thus, such biologically produced hard tissues are considered as superior organic-inorganic hybrid-materials.

Calcium carbonates have three types of crystal structures such as calcite, aragonite, and vaterite. Calcite has a trigonal structure, which is stable at room temperature and normal pressure. On the other hand, aragonite has an orthorhombic structure, which is stable at low temperature and high pressure. Interestingly, shellfishes can concurrently produce these two different crystal structures of calcium carbonates during their life processes. ${ }^{1)}$ However, this mechanism has not yet been elucidated. If the biomineralization mechanism is clarified, it might be possible to create new nano materials by utilizing these unique biological functions and controlling the architecture or interface between two different phases on a nano scale. Therefore, the study of biominerals and/or biomineralization from the viewpoint of materials science is important for both the biological and materials science fields.

The purpose of this work is to investigate the microstructure and orientation distribution of aragonite crystals in the nacreous layer of cultured $P$. penguin, which is a wellknown pearl shell. Figure 1 shows a schematic illustration of the cross section of the ostracum structure of $P$. penguin. ${ }^{2,3)}$

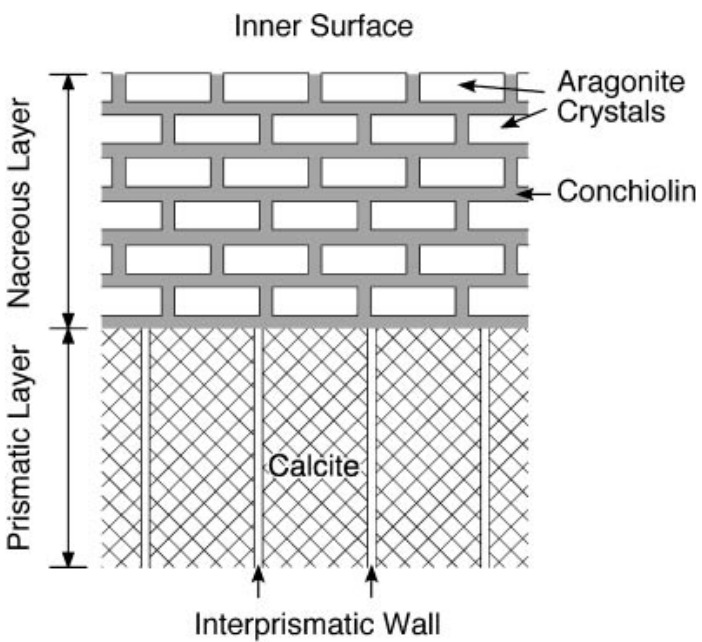

Fig. 1 Schematic illustration of the cross section of the ostracum structure of $P$. penguin near inner surface. ${ }^{2,3)}$

Broadly speaking, its ostracum consists of two layers, i.e., the nacreous layer on the top of the inner shell surface, and the prismatic layer. The nacreous layer, whose structure is basically the same as that of the pearl developed within the shell, is composed of aragonite crystals, as shown in the figure. The quality of pearls will be discussed on the basis of the obtained results.

\section{Experimental Procedure}

Two types of shells of 6-year-old cultured $P$. penguin were provided by Tasaki Shinju Co., Ltd. The first type developed a well-pearlized, commercially valuable pearl, and the other developed a commercially worthless pearl. In this study, the 
former types of shells are referred to as good-quality shells, and the latter are referred to as bad-quality shells. The shells were randomly cut into pieces of about $5 \times 5 \mathrm{~mm}^{2}$ with a low-speed wheel cutter having a diamond blade. The surface microstructure of the nacreous layers was observed using a laser microscope (LM; KEYENCE, VK-9500). The orientation distribution of aragonite crystals in nacreous layers was examined by an X-ray diffractometer (XRD; BRUKER AXS K.K., D8 DISCOVER with GADDS) equipped with a monocapillary, $100 \mu \mathrm{m}$ in diameter, and a two-dimensional position sensitive proportional counter (Hi-STAR) using $\mathrm{Cu}$ $\mathrm{K} \alpha$ radiation at $40 \mathrm{kV}$ and $40 \mathrm{~mA}$. Figure 2 shows the measurement setup and beam alignment for XRD. The outer surface of a piece of the shells is fixed onto the Eulerian cradle stage so that the inner shell surface faces the optical microscope. The pole figures for the (002) and (111) planes were drawn using these two plane diffractions so that the irradiation areas were estimated to be approximately $0.12 \mathrm{~mm}^{2}$ excluding the divergence of the incident X-ray beam. The integral angle ranges are $0.8^{\circ}$ for these two

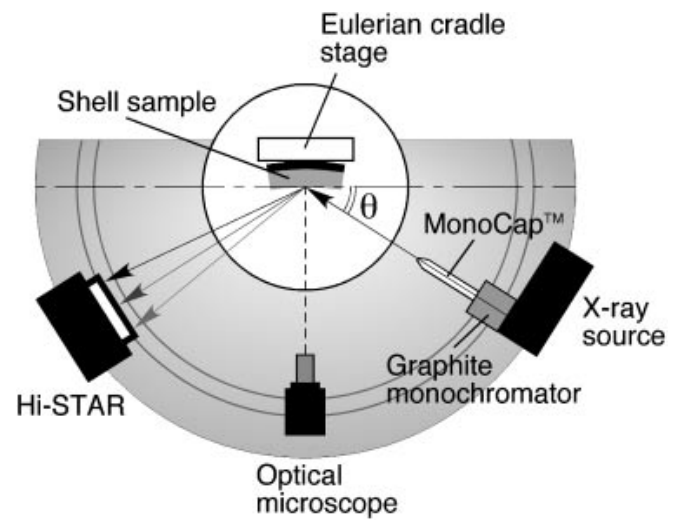

Fig. 2 XRD measurement setup and beam alignment of D8 DISCOVER with GADDS by BRUKER AXS K.K.

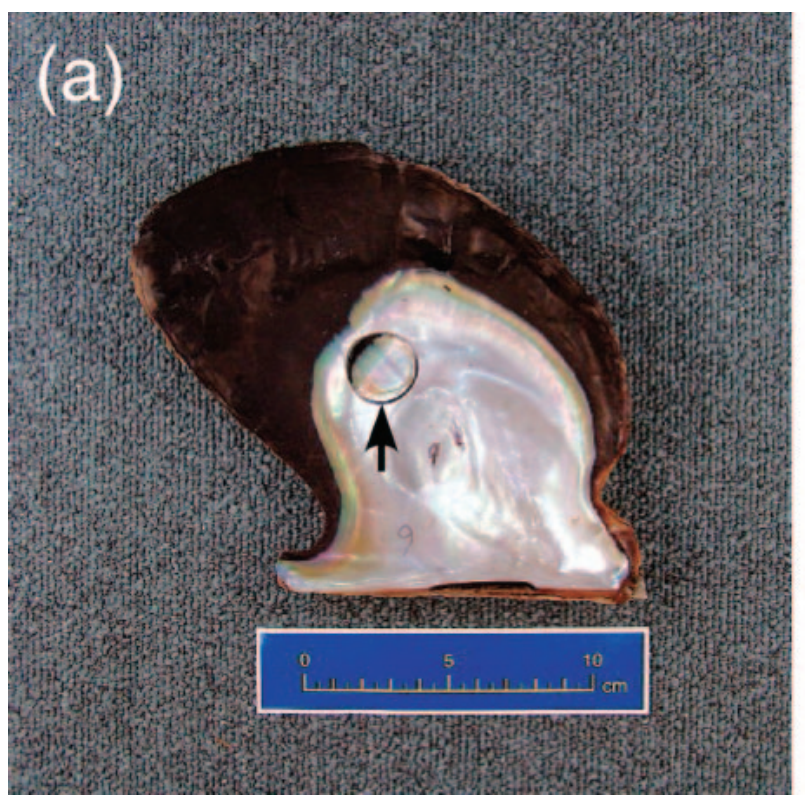

diffractions. Although the shell surfaces have a curvature, its effect on the X-ray diffraction is negligible due to the small beam diameter of this equipment.

\section{Results and Discussion}

Figure 3 shows images of the inner and outer morphology of a good-quality shell. From the inner shell appearance (Fig. $3(\mathrm{a})$ ), it is observed that the nacreous layer (bright layer) is developed onto a prismatic layer (dark layer) on the inner side. A circular pattern marked with an arrow, as seen in Fig. 3(a), reveals the trace of a pearl, which has the same structure as that of the nacreous layer mentioned before. The nacreous structure of aragonite crystals was examined by LM. Figure 4 shows laser micrographs of the surface structure composed of aragonite crystals formed in the two different shells. The shell shown in Figs. 4(a) and (c) represents a good-quality shell, whereas the one shown in Figs. 4(b) and (d) represents a bad-quality shell. The nacreous layer of the good-quality shell exhibits helical patterns at a lower magnification, as shown in Fig. 4(a). When the nacreous layer is observed at a higher magnification (Fig. 4(c)), it is revealed that the helical patterns are drawn by the growth forefronts of the nacreous layer. Aragonite crystals observed at the forefronts are hexagonal in shape and are loosely packed. Some of the aragonite crystals near the forefronts, whose size is evidently smaller than the others, are completely isolated. It should be noted that the observed aragonite crystals seem to be oriented along a specific direction. In the backward areas of the forefronts, aragonite crystals are densely packed. On the other hand, no clear pattern is seen in the nacreous layers of bad-quality shells, as shown in Figs. 4(b) and (d). The shape of the aragonite crystals is also hexagonal, but the crystals seem to be oriented randomly, as seen in Fig. 4(b). These results suggest that the orientation distributions of aragonite crystals in these two shells are different.

Orientation distributions of aragonite crystals were meas-

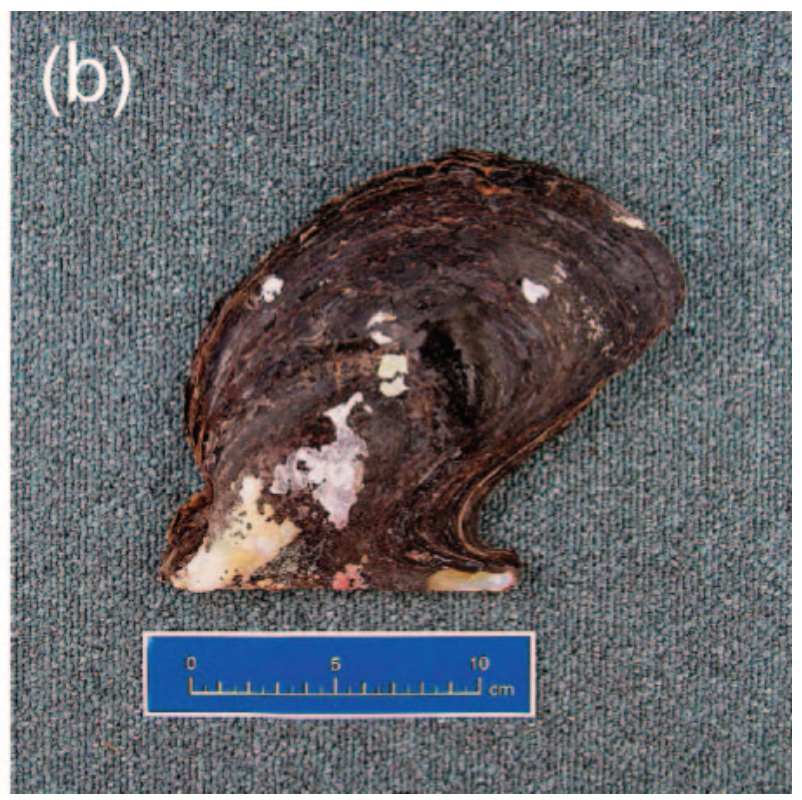

Fig. 3 Images of inner and outer morphology of a good-quality shell. 

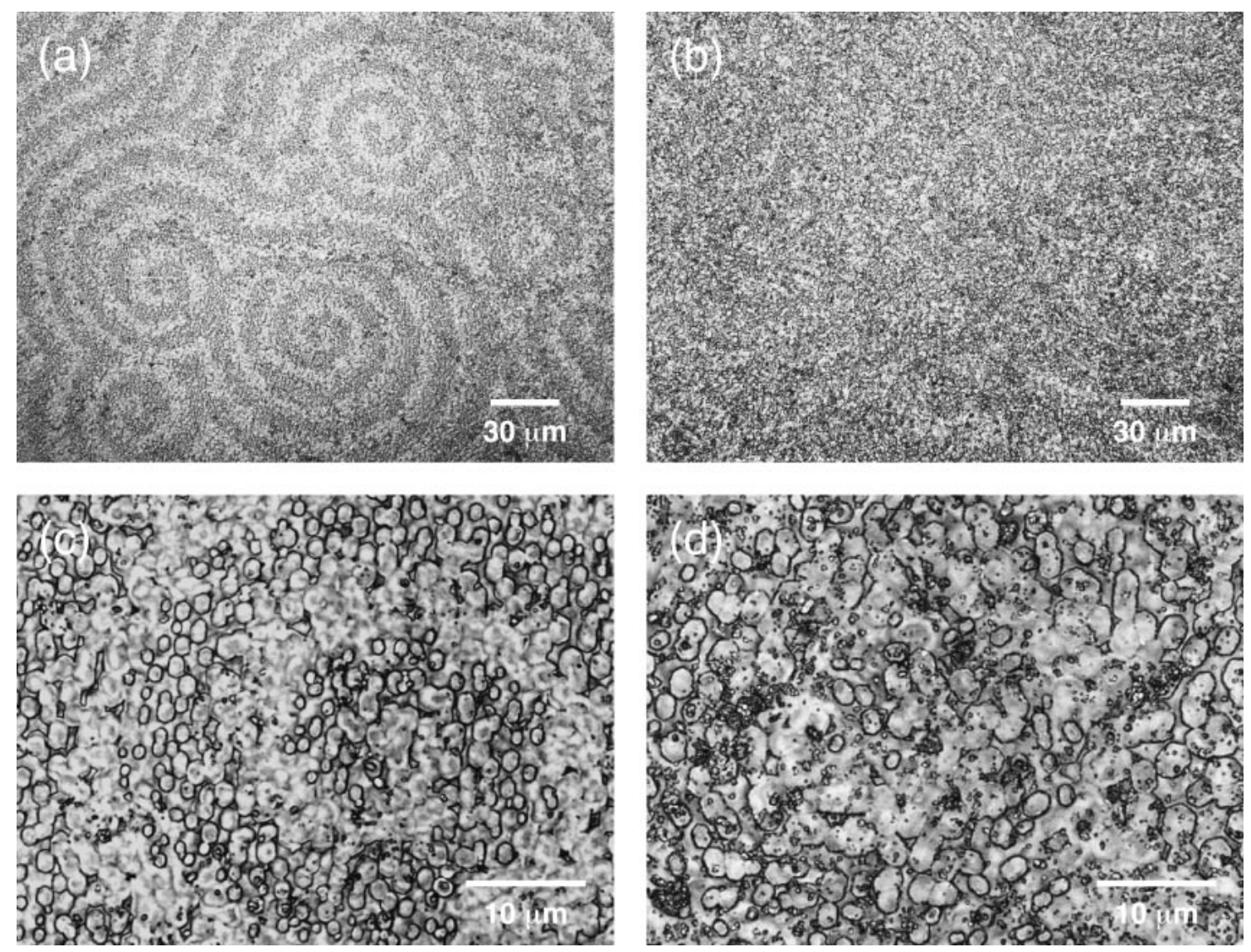

Fig. 4 Laser micrographs of surface microstructures of the nacreous layers in (a) and (c) good- and (b) and (d) bad-quality shells.

ured in the nacreous layers of both shell types by an X-ray diffractometer equipped with a mono-capillary, $100 \mu \mathrm{m}$ in diameter, and a Hi-STAR. Figure 5 shows X-ray Debye rings obtained from the nacreous layer surfaces for the (a) good and (b) bad-quality shells. The ring patterns are significantly different between the two shell types, indicating that the aragonite crystals in the nacreous layers have different orientation distributions.

Figure 6 shows pole figures for the nacreous layer of goodquality shells, which were measured using (002) and (111) reflections of aragonite. The normal directions (ND) of the pole figures are almost parallel to the ND of the inner shell surfaces. The (002) reflection distribution (Fig. 6(a)) clearly displays the texture formation of aragonite crystals around the ND direction, indicating that its basal plane, (001), is strongly oriented toward the normal direction of the inner shell surface in the nacreous layers, as previously reported. ${ }^{4)}$ The $\{111\}$ reflection distribution (Fig. 6(b)) also clearly displays texture formation. Of the six poles, the intensity of two poles paired along a diagonal line is much weaker than that of the others. Compared with the (001) standard projection of the orthorhombic structure of aragonite crystals (Fig. 7), it is clarified that the nacreous layer of the goodquality shell has a strong texture compared to that of the basal planes of aragonite crystals that face the inner shell surface (i.e., the $c$ axis is parallel to the normal direction of the inner shell surface); moreover their [100] and [010] axes (i.e., the $\boldsymbol{a}$ and $\boldsymbol{b}$ axes) are oriented toward almost the same direction, respectively. Unfortunately, since the shell specimens were randomly cut from the shells, it is unclear here, which directions in the shell shown in Fig. 3(a) correspond to directions in those pole figures. The weaker paired poles are located approximately $\pm 60^{\circ}$ away from the stronger poles around the $c$ axis. The reason that the weaker paired poles exist in the $\{111\}$ pole figure will be discussed.

Figure 8 shows the pole figures for the nacreous layer of the bad-quality shell measured in the same way as the goodquality shell. Their NDs are also close to the ND of the inner shell surfaces. The (002) reflection distribution (Fig. 8(a)) is almost the same as that for the good-quality shell (Fig. 6(a)). This indicates that even in the bad-quality shell, the basal planes of aragonite crystals in the nacreous layer face the inner shell surface. On the contrary, the $\{111\}$ reflection distribution displays a ring pattern, as shown in Fig. 8(b). These results indicate that the $\boldsymbol{a}$ and $\boldsymbol{b}$ axes of aragonite crystals are randomly oriented about the $c$ axis in the nacreous layer of the bad-quality shell. This random orientation distribution induces irregular reflection of light near the surfaces. This may be the reason that the quality of pearls developed in the bad-quality shells was not good.

Then, the question arises - "Why is the orientation distribution of aragonite crystals in the nacreous layer different between the two types of the shells?" The clue to answering this question may be found in the helical pattern formation of aragonite crystals as seen in Figs. 4(a) and (c). The thickness of aragonite crystals was reported to be from 300 to $600 \mathrm{~nm}$ for $P$. penguin, ${ }^{6}$ ) which almost corresponds to the wavelength range of the visible light. In general, the top 

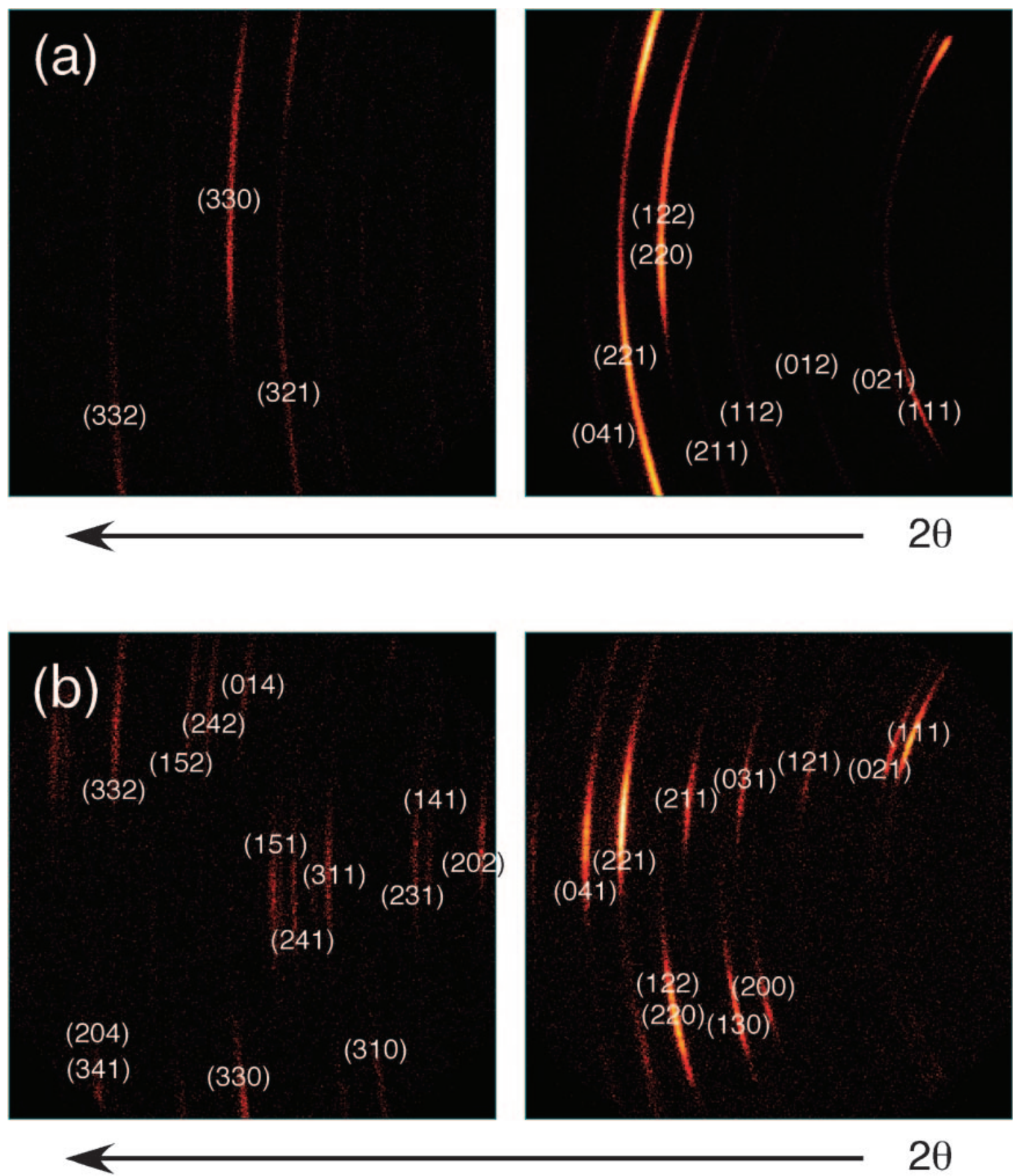

Fig. 5 X-ray Debye ring patterns obtained from the nacreous layers of (a) good- and (b) bad-quality shells.

and bottom faces of aragonite crystals in nacreous layer of shells are parallel to the $\{001\}$ basal plane. ${ }^{4)}$ Therefore, the strong texture in which the $c$ axes are aligned is developed in the nacreous layers, as seen in Figs. 6(a) and 7(a). On the other hand, several crystallographic planes such as $\{110\}$, (100), and (010) were reported to appear on the side faces of the aragonite crystals. ${ }^{4}$ Figure 9 shows schematic illustrations of the predicted shapes of an aragonite crystal as viewed from the $c$ axis. Since all six corners of the hexagonal crystals have obtuse angles (Fig. 4, it is considered that the side faces of aragonite crystals in the nacreous layers are formed with the $\{110\}$ planes and the (010) plane, as shown in Fig. 9(b). An interesting point is that the angles of the six corners of the hexagon are close to $120^{\circ}$. If the lengths of the six edges are almost the same in this hexagon, the shape would allow aragonite crystals to be closely packed even though the crystals are rotated about the $c$ axis by $\pm 60^{\circ}$. Such a graphoepitaxy-like growth process may occur in the nacreous layer of the good-quality shells. Figure 6(b) shows that the weaker paired poles of the $\{111\}$ reflection is located at $\pm 60^{\circ}$ away from the stronger paired poles about the $c$ axis. This evidence supports the above idea. If the crystals can be closely packed, it would be possible to develop the nacreous layer in a similar manner as the crystal growth along screw dislocations. However, isolated crystals near the growth forefronts are also observed in Fig. 4(c), which seem to be oriented along the same direction as that of the others. An epitaxy-like growth process may also occur in the nacreous 
(a) (002)

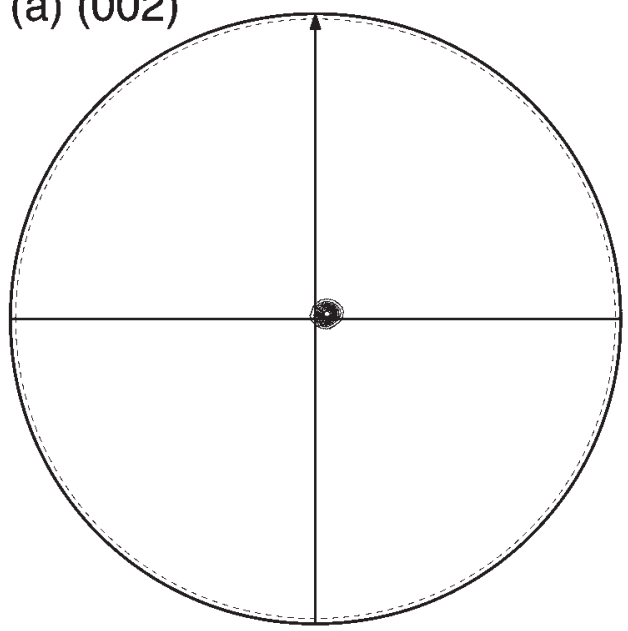

(b) (111)

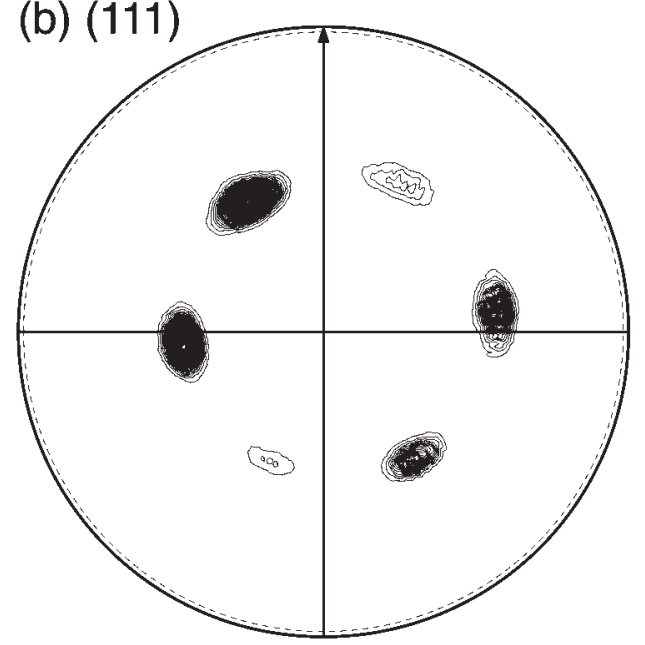

Fig. 6 Pole figures for the nacreous layer of a good-quality shell obtained using (a) (001) and (b) (111) reflections of aragonite.
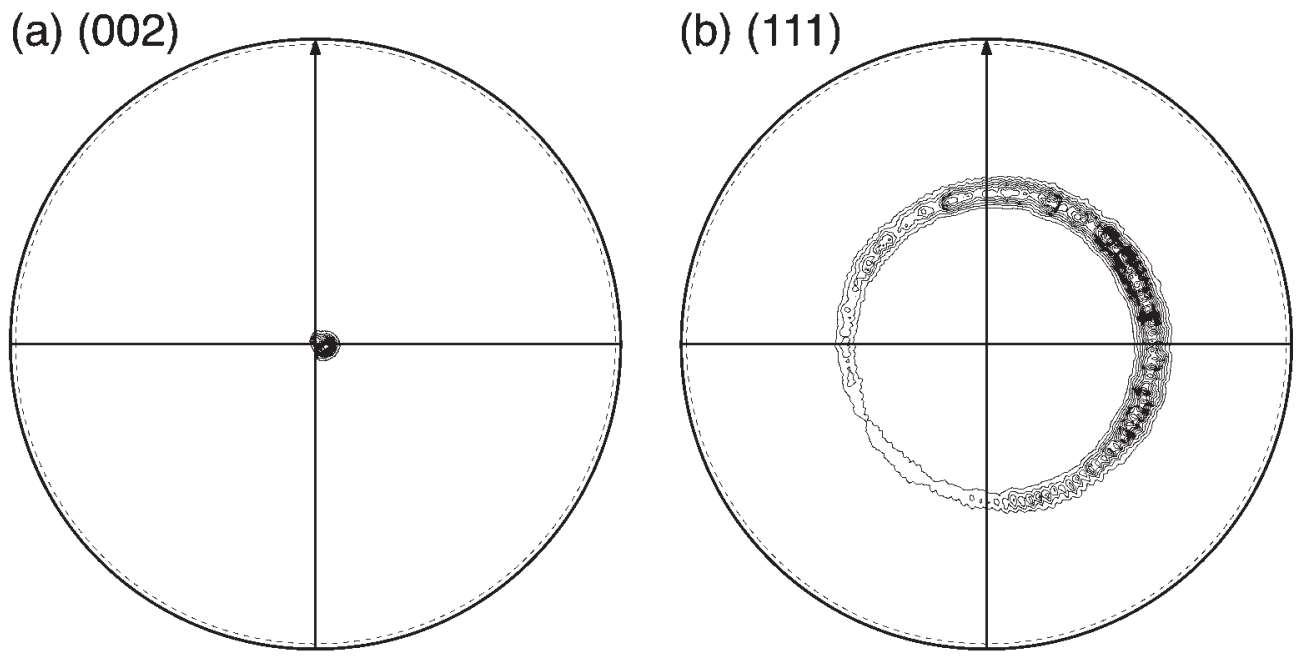

Fig. 8 Pole figures of the nacreous layer of a bad-quality shell obtained using (a) (001) and (b) (111) reflections.

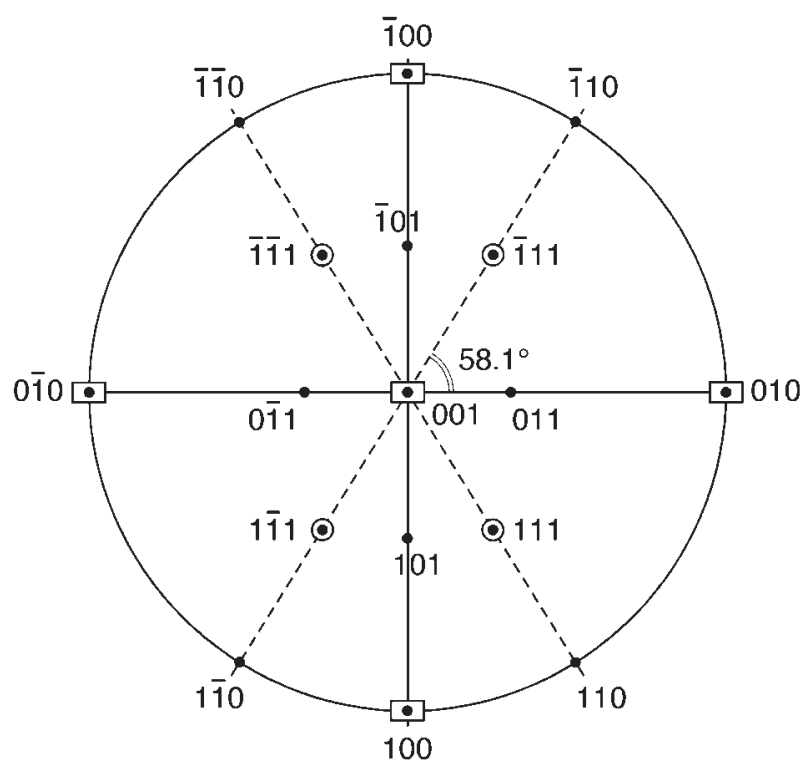

Fig. 7 The (001) standard projection of the orthorhombic structure of aragonite crystals. $a=0.494 \mathrm{~nm}, b=0.794 \mathrm{~nm}$ and $c=0.572 \mathrm{~nm}^{5}$ ) layer of good-quality shells. As mentioned in the introduction, aragonite crystals are bound by protein complexes called "interlamellar matrix" and "intercrystalline matrix" in the nacreous layer. In $P$. penguin as well as other pearl shells, the roll played by protein complexes in the orientation distribution of aragonite crystals in nacreous layer is still not understood. Further studies are required to clarify this mechanism of the development of nacreous layer in pearl shells.

\section{Conclusions}

This paper investigated the microstructure and orientation distribution of aragonite crystals in the nacreous layer of cultured $P$. penguin, a well-known pearl shell. The conclusions obtained are as follows:

(1) Helical patterns drawn by the growth forefronts on the nacreous layer were observed for good-quality shells, whereas no clear pattern was observed on the nacreous layer surfaces of bad-quality shells.

(2) The aragonite crystals in the nacreous layers are plateshaped and hexagonal for both good and bad-quality 
(a)

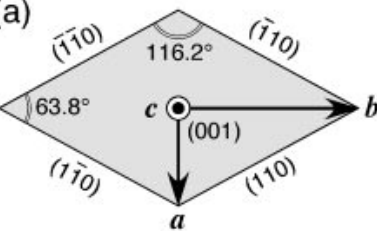

(c)

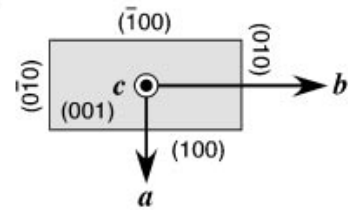

(b)

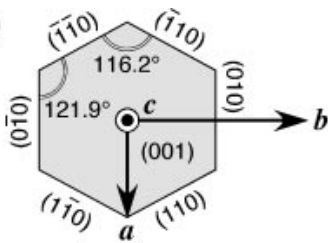

(d)

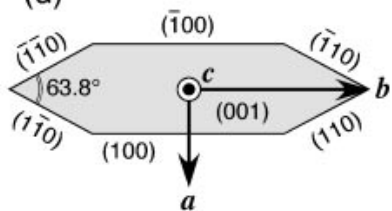

Fig. 9 Schematic illustrations of the predicted shapes of an aragonite crystal viewed from the $c$ axis. $a=0.494 \mathrm{~nm}, b=0.794 \mathrm{~nm}$ and $c=0.572 \mathrm{~nm}^{5)}$

shells. It is considered that the top and bottom faces of the aragonite crystals are parallel to the (001) plane and the side faces the $\{110\}$ and (010) planes.

(3) Isolated aragonite crystals are observed near the growth forefronts on the nacreous layer surfaces of goodquality shells, which are smaller than those inside the forefronts. The aragonite crystals in the nacreous layer of good-quality shells seem to be harmonically oriented along a direction irrespective of the extent of packing and isolation. The aragonite crystals of bad-quality shells seem to be randomly oriented in the nacreous layer, compared with those of the good-quality shells.

(4) The nacreous layer of good-quality shells has a strong texture of aragonite crystals. Basically, the (001) basal

planes are parallel to the inner shell surface, and the [100] and [010] axes are oriented in almost the same direction, respectively. Some of the aragonite crystals are rotated around the $c$ axis by approximately $\pm 60^{\circ}$ from the basic orientation distribution. The nacreous layer of bad-quality shells also has a texture. Their (001) basal planes are parallel to the inner shell surface in a similar manner as those of the good-quality shells, but their [100] and [010] axes are randomly oriented about the $c$ axis.

\section{Acknowledgments}

The authors thank N. Kimura and M. Anraku of Tasaki Shinju Co., Ltd., for providing the shells of cultured $P$. penguin. Thanks are also due to T. Kurosawa and N. Yamada of BRUKER AXS K.K. for the XRD measurements and T. Kuwabara of KEYENCE Corp. for the LM observations. This work is partly supported by the Grant-in-Aid for Science Research on Priority Area (No. 14658279) from the Ministry of Education, Science and Culture of Japan.

\section{REFERENCES}

1) K. Wada: Shinju no Kagaku, (Shinju Shinbun, Tokyo, 1999) pp. 13-25.

2) A. Matsushiro: Chemistry Today 383 (2003) 32-38.

3) K. Yoshimi, M. Shoji, A. Yamauchi, T. Ogawa, T. Naganuma and K. Muramoto: Boundary 19 (2003) 8-11.

4) K. Wada: J. Jpn Ass. Crystal Growth 12 (1985) 57-70.

5) K. Wada: Shinju no Kagaku, (Shinju Shinbun, Tokyo, 1999) pp. 135.

6) K. Wada: The Mechanisms of Biomineralization in Animals and Plants, ed. by M. Omori and N. Watabe, (Tokai Univ. Press, 1980) 79-92. 